\title{
Impact of a Pharmacist-driven Methicillin- resistant Staphylococcus aureus Polymerase Chain Reaction Nasal Swab Protocol on the De- escalation of Empiric Vancomycin in Patients with Pneumonia in a Rural Healthcare Setting
}

Precious Dadzie ${ }^{1}$, Tyson Dietrich ${ }^{2}$, John Ashurst ${ }^{3}$

1. Pharmacy, Kingman Regional Medical Center, Kingman, USA 2. Pharmacy / Infectious Diseases, Kingman Regional Medical Center, Kingman, USA 3. Emergency Medicine, Kingman Regional Medical Center, Kingman, USA

Corresponding author: John Ashurst, ashurst.john.32.research@gmail.com

\section{Abstract}

\section{Introduction}

Pneumonia caused by methicillin-resistant Staphylococcus aureus (MRSA) carries a high rate of morbidity and mortality. Many clinicians empirically treat those at risk of developing MRSA pneumonia with vancomycin. Several studies have identified a high negative predictive value of the MRSA polymerase chain reaction (PCR) nasal swab test in lower respiratory tract infections, suggesting it can be used to guide the de-escalation of empiric anti-MRSA therapy.

\section{Objective}

To evaluate the impact of a pharmacist-driven MRSA PCR nasal swab protocol on the de-escalation of empiric vancomycin in patients with pneumonia in a rural healthcare setting. Secondarily, to assess the rate of hospital length of stay, the rate of vancomycin-associated acute kidney injury, and in-hospital mortality after pharmacist-driven de-escalation of empiric vancomycin in patients with pneumonia.

\section{Methods}

A retrospective, single-center, pre-post cohort study was conducted in patients after the implementation of a pharmacist-driven protocol allowing pharmacists to obtain nasal swabs and PCR testing for MRSA in those on empiric vancomycin therapy for suspected MRSA pneumonia. Based on negative test results, pharmacists recommended a de-escalation of empiric vancomycin to the physician. Patients were included if they were adults at least 18 years of age, had a physician diagnosis of suspected or confirmed pneumonia, and initiated on at least one dose of intravenous vancomycin within 48 hours of admission.

Received 10/26/2019

Review began 11/10/2019 Review ended 12/10/2019 Published 12/13/2019

๑) Copyright 2019 Dadzie et al. This is an open access article distributed under the terms of the Creative Commons Attribution License CC-BY 3.0., which permits unrestricted use, distribution, and reproduction in any medium, provided the original author and source are credited.

\section{Results}

A total of 79 patients were identified for inclusion in the pre-protocol group $(n=32)$ or post-protocol group $(n=47)$. The mean duration of vancomycin therapy in the pre-protocol group was 3.1 days as compared to 1.7 days in the post-protocol group for a 1.4 days reduction $(p=0.044)$. There was no significant impact on the number of vancomycin cases de-escalated within 24 hours $(\mathrm{p}=0.14)$ but there was a significant reduction at 48 hours ( $p=0.01$ ). Protocol implementation was associated with a reduction in the average length of hospitalization ( 8 versus 5.20 days, $\mathrm{p}=0.006$ ). Neither group had a vancomycin-associated acute kidney injury or in-hospital mortality.

\section{Conclusion}

Among patients with suspected MRSA pneumonia, a pharmacist-driven MRSA PCR nasal swab protocol resulted in a significant reduction of empiric vancomycin duration of therapy without an adverse impact on clinical outcomes in a rural healthcare setting.

Categories: Internal Medicine, Infectious Disease

Keywords: mrsa, antibiotic stewardship, pneumonia

\section{Introduction}

Pneumonia has been credited as one of the leading causes of infectious disease-related deaths in the United States [1]. Staphylococcus aureus was the second most common pathogen noted in all health-care-associated infections in the United States between 2011 and 2014 [2]. Although not a common pathogen seen in community-acquired pneumonia, methicillin-resistant Staphylococcus aureus (MRSA) is an emerging 
pathogen that accounts for between $20 \%$ and $40 \%$ of all nosocomial infections [3-4]. Current guidelines from the American Thoracic Society and Infectious Diseases Society of America (IDSA) recommend empiric treatment for those at risk of developing MRSA pneumonia despite its low prevalence in the community setting [5-6].

MRSA is a common pathogen that has been known to colonize the nares of patients and assay-confirmed infection has been shown to be predictive for the development of future MRSA infections [7]. The MRSA polymerase chain reaction (PCR) assay is a readily available test that carries a negative predictive value (NPV) of between $95 \$$ and $99 \%$ for the detection of MRSA [8-9]. Data have shown that in the absence of nares, MRSA has been a negative predictor for the development of MRSA pneumonia [10]. The objective of this study was to evaluate the impact of a pharmacist-driven MRSA PCR nasal swab protocol on the deescalation of empiric vancomycin in patients with pneumonia in a rural healthcare setting. The secondary objective was to assess the rate of hospital length of stay, rate of vancomycin-associated acute kidney injury, and in-hospital mortality after pharmacist-driven de-escalation of empiric vancomycin in patients with pneumonia.

\section{Materials And Methods Study design and patient selection}

This retrospective, single-center, pre-post cohort study was conducted at a 235-bed hospital in rural Arizona, with an average annual emergency department volume of 50,000 patients. The pre-protocol group consisted of patients admitted from June 1, 2018, through September 30, 2018, and the post-protocol group consisted of patients admitted between November 1, 2018, and January 31, 2019. Adult patients at least 18 years of age with a physician diagnosis of pneumonia within 48 hours of admission and initiated on at least one dose of intravenous (IV) vancomycin for empiric MRSA coverage were included in the study. All patients included in the study had the diagnosis of pneumonia and were excluded if they were pregnant, admitted to the ICU, or received IV vancomycin for a concomitant infection. Additional exclusion criteria included if the nasal swab test was performed more than one month prior to the respiratory culture for patients admitted from the outpatient department, more than seven days prior to the respiratory culture for hospital-acquired cases, and if the nasal swab test was performed more than three days after the respiratory culture was collected. Vancomycin-associated nephrotoxicity was defined as a rise in serum creatinine of $0.5 \mathrm{mg} / \mathrm{dl}$ or $50 \%$ above baseline on at least two consecutive measurements after 72 hours of vancomycin and with no other apparent cause.

\section{Protocol implementation}

An MRSA PCR nasal swab protocol was developed as part of an antimicrobial stewardship initiative, and education regarding the protocol and clinical utility of the MRSA PCR nasal swab test was provided to all affected healthcare providers during October 2018. Hospitalists were educated individually and nurses received education via email communication. Education was provided to the pharmacist in multiple settings, including department meetings, individual interactions, and via email communication. The protocol was implemented on November 1, 2018, and authorized pharmacists to order an MRSA PCR nasal swab test for patients with pneumonia started on IV vancomycin. If the results of the swab were negative, the pharmacist would contact the provider to discuss the discontinuation of vancomycin. The ultimate decision to de-escalate vancomycin remained with the physician. Pharmacists and hospitalists received re-education throughout the study.

\section{Statistical analysis}

A sample size of 24 patients in each group was required to detect a one-day reduction in the duration of vancomycin therapy between groups, assuming an average baseline of $4+1.5$ days. Categorical data were analyzed using the chi-squared test and continuous data were analyzed using the t-test. All tests were twotailed, with statistical significance set at a p-value of less than 0.05 .

\section{Results}

In total, 888 patient records were reviewed and 79 patients were included in the final analysis. Overall, the baseline demographic data and clinical characteristics were similar in the pre- and post-protocol groups (Table 1). 


\section{Cureus}

\begin{tabular}{|c|c|c|}
\hline Characteristics & Pre-protocol $(n=32)$ & Post-protocol $(n=47)$ \\
\hline Median age (year) & $69(28-81)$ & $71(30-90)$ \\
\hline Male & $16(50 \%)$ & $32(68.1 \%)$ \\
\hline Congestive Heart Failure & $7(21.9 \%)$ & $12(25.5 \%)$ \\
\hline Diabetes Mellitus, Type 2 & $12(37.5 \%)$ & $20(42.6 \%)$ \\
\hline Chronic Obstructive Pulmonary Disease & $11(34.4 \%)$ & $19(40.4 \%)$ \\
\hline Immunosuppression & $1(3.1 \%)$ & 0 \\
\hline Nursing Home/Extended Care Facility & $6(18.8 \%)$ & $5(10.6 \%)$ \\
\hline Recent Hospitalization & $12(37.5 \%)$ & $17(36.2 \%)$ \\
\hline
\end{tabular}

TABLE 1: Patient demographics

There was no statistically significant difference in the number of vancomycin de-escalated patients within 24 hours between the pre- and post-groups ( $34 \%$ vs $51 \%$; $p=0.14$ ). However, there was a significant difference in the amount of vancomycin de-escalation at 48 hours between the two groups (63\% vs $87 \%$; $\mathrm{p}=0.01$ ). The duration of empiric vancomycin therapy was reduced by 1.4 days per patient in those with negative MRSA PCR swabs ( 3.1 days vs 1.7 days; $\mathrm{p}=0.04$ ). Length of stay was also reduced by 2.8 days following the implementation of the protocol $(\mathrm{p}=0.006)$. There was no difference in the number of vancomycin-associated acute kidney injury or in-hospital mortality.

\section{Discussion}

The overuse of anti-MRSA antibiotics has been associated with increased antibiotic resistance, nephrotoxicity, and increased healthcare costs and should be de-escalated once negative culture results are obtained [11]. Much like previous research, a pharmacy-driven protocol utilizing an MRSA nasal swab PCR in those suspected of having MRSA pneumonia resulted in a shorter duration of vancomycin therapy without increasing adverse events [11-12]. One could postulate that by decreasing the number of days a patient is on vancomycin for MRSA coverage in pneumonia that total healthcare costs would also be decreased because of fewer days in the hospital and less serum vancomycin levels being obtained. According to Smith et al., a \$108 reduction in cost per patient was noted in medication-related costs following the implementation of a similar protocol [13].

Although a statistically significant number of vancomycin de-escalations was noted at 48 hours following a negative MRSA nasal PCR, a large number of cases could have been de-escalated sooner. This data coincides with previous data that protocol compliance has notably been poor for a pharmacy-driven protocol utilizing an MRSA nasal swab PCR to de-escalate vancomycin in pneumonia [11]. One would have imagined a larger number of de-escalations at 24 hours due to the Hawthorne effect but this was not seen till 48 hours. The exact mechanism for these findings is unclear but could be related to the ability to overcome previous practice habits [14].

The single-center retrospective nature of this study with a lack of randomization decreases external validity. This study had a relatively small sample size; however, this did not have a significant impact on outcomes. Another limitation was pharmacy staff compliance with protocol requirements was not evaluated. Despite evaluation, there were occasions when the MRSA PCR nasal swab test was not ordered, with a STAT priority resulting in a delay of the test result, which delayed potential recommendation for de-escalation.

\section{Conclusions}

The implementation of a pharmacist-driven MRSA PCR nasal swab protocol had a significant impact on empiric vancomycin utilization in patients with pneumonia. No difference was seen in the rate of vancomycin-associated acute kidney injury or in-hospital mortality after pharmacist-driven de-escalation of empiric vancomycin in patients with pneumonia. Future studies should be aimed at continuing to validate the use of MRSA PCR nasal swabs for the de-escalation of empiric MRSA coverage in patients diagnosed with pneumonia. Missing in the already growing body evidence for this practice are multicentered, randomized controlled trials comparing MRSA PCR nasal swabs to traditional standards of practice.

\section{Additional Information}

\section{Disclosures}


Human subjects: Consent was obtained by all participants in this study. Kingman Regional Medical Center issued approval 0160. The study was approved by the Kingman Regional Medical Center Institutional Review Board. Animal subjects: All authors have confirmed that this study did not involve animal subjects or tissue. Conflicts of interest: In compliance with the ICMJE uniform disclosure form, all authors declare the following: Payment/services info: All authors have declared that no financial support was received from any organization for the submitted work. Financial relationships: All authors have declared that they have no financial relationships at present or within the previous three years with any organizations that might have an interest in the submitted work. Other relationships: All authors have declared that there are no other relationships or activities that could appear to have influenced the submitted work.

\section{References}

1. Murphy SL, Xu J, Kochanek KD, Curtin SC, Arias E: Deaths: final data for 2015. Natl Vital Stat Rep. 2017, 66:1-75.

2. Weiner LM, Webb AK, Limbago B, et al.: Anti-microbial resistant pathogens associated with healthcareassociated infections: summary of data reported to the National Healthcare Safety Network at the Centers for the Disease Control and Prevention, 2011-2014. Infect Control Hosp Epidemiol. 2016, 37:1288-1301. 10.1017/ice.2016.174

3. Rubinstein E, Kollef MH, Nathwani D: Pneumonia caused by methicillin-resistant Staphylococcus aureus . Clin Infect Dis. 2008, 46:378-385. 10.1086/533594

4. Shorr AF, Tabak YP, Gupta V, Johannes RS, Liu LZ, Kollef MH: Morbidity and cost burden of methicillin resistant Staphylococcus aureus in early onset ventilator associated pneumonia. Crit Care. 2006, 10:97. $10.1186 /$ cc4934

5. Kalil AC, Metersky ML, Klompas M, et al.: Management of adults with hospital-acquired and ventilatorassociated pneumonia: 2016 clinical practice guidelines by the Infectious Diseases Society of America and the American Thoracic Society. Clin Infect Dis. 2016, 63:61. 10.1093/cid/ciw353

6. Mandell LA, Wunderink RG, Anzueto A, et al.: Infectious Diseases Society of America/ American Thoracic Society consensus guidelines on the management of community-acquired pneumonia in adults. Clin Infect Dis. 2007, 44:27-72. 10.1086/511159

7. Davis KA, Steward JJ, Crouch HK, Florez CE, Hospenthal DR: Methicillin-resistant Staphylococcus aureus nares colonization at hospital admission and its effects on subsequent MRSA infection. Clin Infect Dis. 2004, 39:776-782. 10.1086/422997

8. Dangerfield B, Chung A, Webb B, Seville MT: Predictive value of methicillin-resistant Staphylococcus aureus nasal swab PCR assay for MRSA pneumonia. Antimicrob Agents Chemother. 2014, 58:859-864. 10.1128/AAC.01805-13

9. Robicsek A, Suseno M, Beaumont J, Thomson RB Jr, Peterson LR: Prediction of methicillin-resistant Staphylococcus aureus involvement in disease sites by concomitant nasal sampling. J Clin Microbiol. 2008, 46:588-592. 10.1128/TCM.01746-07

10. Parente DM, Cunha BC, Mylonakis E, Timbrook TT: The clinical utility of methicillin-resistant Staphylococcus aureus (MRSA) nasal screening to rule out MRSA pneumonia: a diagnostic meta-analysis with antimicrobial stewardship implications. Clin Infect Dis. 2018, 67:1-7. 10.1093/cid/ciy024

11. Dunaway S, Orwig K, Arbogast Z, Myers ZL, Sizemore JA, Giancola SE: Evaluation of a pharmacy-driven methicillin-resistant staphylococcus aureus surveillance protocol in pneumonia. Int J Clin Pharm. 2018, 40:526-532. 10.1007/s11096-018-0647-3

12. Willis C, Allen B, Tucker C, Rottman K, Epps K: Impact of a pharmacist-driven methicillin resistant staphylococcus aureus surveillance protocol. Am J Health Syst Pharm. 2017, 74:1765-1773.

13. Smith MN, Erdman MJ, Ferreira JA, Aldridge P, Jankowski CA: Clinical utility of methicillin-resistant Staphylococcus aureus nasal polymerase chain reaction assay in critically ill patients with nosocomial pneumonia. J Crit Care. 2017, 38:168-171. 10.1016/j.jcrc.2016.11.008

14. Cabana M, Rand CS, Powe NR, Wu AW, Wilson MH, Abboud PA, Rubin HR: Why don't physicians follow clinical practice guidelines: a framework for improvement. JAMA. 1999, 282:1458-1465. 10.1001/jama.282.15.1458 